\title{
Temperature Calculation of Bare and Insulated Steel Structures Exposed to Standard ISO 834 Fire Test
}

\author{
O. KESKI-RAHKONEN
}

Fire Technology Laboratory

Technical Research Centre of Finland

SF-02150 Espoo, Finland

\section{ABSTPACT}

Closed form analytical solutions are obtained for the temperature of bare and insulated steel members in one-dimensional geometry in case the outer surface is exposed to standard ISO 834 fire test. The heat transfer from fire gases into the wall is described by a linearized boundary condition. The thermal material parameters are assumed independent of temperature.

It is shown that the solution of the approximated equation for insulated steel is closely related with that of bare steel. Temperatures are presented as a one-parameter family of curves. Methods to determine. steel temperatures and insulation thicknesses are explained by examples.

\section{INTRODUCTION}

Recently there has appeared several notes [2 - 4$]$ commenting on the temperature calculation methods of fire exposed steel structures as recomnended by various steel design manuals or national fire codes. The purpose of the present note is to add a few features to the discussion by presenting analytical, closed form formulas as solutions of linearized, constant coefficient heat conduction equation. For the heating of bare steel under ISO 834 fire test the solution of the linearized equation is an analytical function of one parameter. Although the 1 inearization is rough, the solution plotted as a family of temperature curves, gives a quick overview of the problem.

For the heating of insulated steel under ISO 834 fire test, the partial differential equation of heat conduction can be approximately replaced by a two parameter differential equation [3]. It is shown here for the first time to our knowledge, that the solution of this approximate equation is almost the same analytical function as for the bare steel. The difference caused by the second parameter allows for an easy geometrical interpretation. 
Consider a planar wall with an insulation layer of thickness $d$ and a steel core as shown in Fig. 1. The heat capacity of the steel per unit area is $Q^{\prime \prime}$. The insulation is described by temperature independent density $p$, specific heat capacity $c$, and thermal conductivity $\lambda$. The left hand side of the insulation layer (fig. 1) is exposed to ISO 834 standard fire with a temperature time dependence

$T_{f}(t)=T_{c} \ln \left(1+t / t_{0}\right)$

where in SI-units $T_{C}=149.83 \mathrm{~K}, t_{0}=7.5 \mathrm{~s}$, and $t$ is expressed in seconds. (Eqn. (1) is the same curve defined in ISO 834 in terms of $1 \mathrm{~g}$ function, but it was transformed here to suit better theoretical purposes. Likewise, SI-units are preferred to any engineering type choices.)

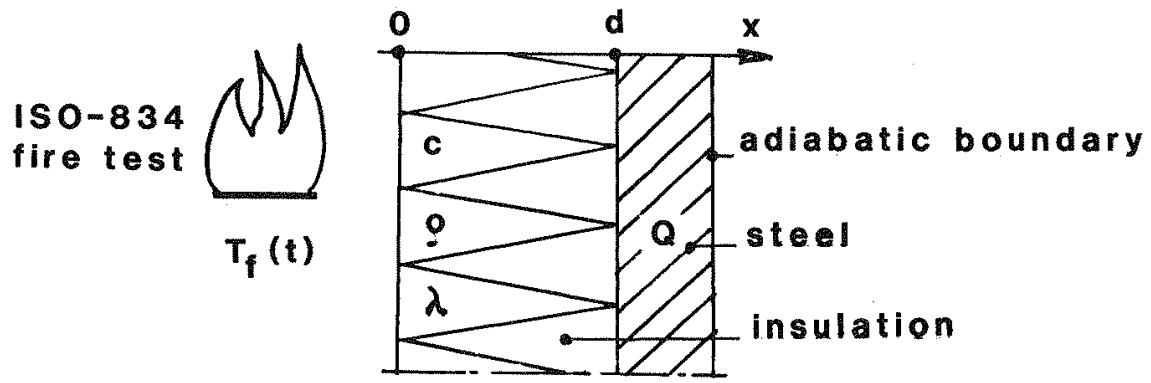

FIGURE 1. Thermal model of an insulated planar steel structure. The steel of heat capacity $Q^{\prime \prime}$ has an adiabatic boundary on the right side and a perfect thermal contact with insulation on the left side. The insulation with thickness $d$, density $\rho$, specific heat capacity $c$, and thermal conductivity $\lambda$ is exposed to standard ISO 834 fire test on the left side.

Setting the coordinate $x$-axis perpendicular to the wall as shown in fig. 1 the temperature $T(x, t)$ in the insulation is given by the heat conduction equation

$\alpha \frac{\partial^{2} T}{\partial x^{2}}=\frac{\partial T}{\partial t}$

where thermal diffusivity $\alpha$

$\alpha=N / \rho$

For the initial condition we take

$T(x, 0)=0$ 
where in the linear system we assumed the environmental temperature $T_{0}=$ 0 . The same result is obtained, if temperatures $T$ are interpreted as differences relative to $T_{0}$. The boundary condition at $x=0$ is given by

$-\lambda \frac{\partial T(0, t)}{\partial x}+h T(0, t)=h T_{f}$

and at $x=d$ by

$\lambda \frac{\partial T(d, t)}{\partial x}+0^{\prime \prime} \frac{\partial T(d, t)}{\partial t}=0$

In eqn. (5) the radiative part of heat transfer is proportinal to the fourth power of the absolute temperature. We linearize the equation at the maximum temperature. Therefore $n$ will be an effective value including convective and radiative contributions calculated as depicted in ref. $[6]$.

When the thickness $d$ of the insulation approaches zero ean. (2) looses its meaning. The heat flux terms on the left hand side in eqns. (5) and (6) approach each other

$\lim _{d \rightarrow 0}\left\{\lambda \frac{\partial T(d, t)}{\partial x}\right\} \rightarrow \lambda \frac{\partial T(0, t)}{\partial x}$ equation

Combining boundary conditions eqns. (5) and (6) yields a single

$Q^{\prime \prime} \frac{d T(t)}{d t}=h\left[T_{f}-T(t)\right]$

or denoting for shortness $h / 0^{\prime \prime}=A$ gives

$\frac{d T(t)}{d t}+A T(t)=A T_{f}$

Eqn. (8) together with the initial condition, eqn. (4), is a first order differential equation which is easily solved in closed form for the Iso 834 standard exposure curve. Eqns. (2) through (6) define mathematically the temperatures of insulation and steel in terms of a partial differential equation the solution of which is much more involved. ECCS remcommendations [7] give two approximate differential equations of type

$\frac{d T(t)}{d t}+A T(t)=A T_{f}(t)-B \frac{d T_{f}(t)}{d t}$

which for proper choices of constant $A$ and $B$ should approximate eqns.

(2) through (6) rather well for times not too short. Constants $A$ and $B$ are defined in terms of steel and insulation parameters [3]

$A=\left(N \alpha / d^{2}\right) /(1+1 / \gamma)(1+N J)$

$B=b /(1+N J)$

where

$J=\frac{\rho_{s} C_{s}^{A} s / H_{p}}{\rho c d}$

and 
Steel parameters are density $\rho_{s}$, specific heat capacity $\mathrm{C}_{S}$ and section factor $H_{p} / A_{S}$. In eans. (10) and (11) b and $\mathrm{N}$ are adjustable weighting factors. Melinek and Thomas [3] have shown that optimal choices of these for long time are as follows:

$b=\frac{J\left(\frac{1}{2}+\frac{2}{3 \gamma}\right)+\frac{1}{8}+\frac{1}{2 \gamma}}{J\left(1+\frac{3}{\gamma}+\frac{3}{\gamma^{2}}\right)+\frac{5}{8}+\frac{5}{2 \gamma}+\frac{3}{\gamma^{2}}}$

and

$N=2(b+1) \frac{\left(1+\frac{1}{\gamma}\right)}{\left(1+\frac{2}{\gamma}\right)}$

We explain in the numerical example later how to determine iteratively constants $A$ and $B$ using eqns. (10) - (15).

HEATING OF BARE STEEL

The general solution of eqn. ( $\left.8^{\prime}\right)$ with initial conditions $T(0)=T_{f}(0)=0$ is

$T(t)=A_{0} f^{t} \exp \left[A\left(t^{\prime}-t\right)\right] T_{f}\left(t^{\prime}\right) d t^{\prime}$

or in more transparent form obtained from ean. (16) integrating by parts

$T(t)=T_{f}(t)-{ }_{0}^{\int} t^{t} \exp \left[A\left(t^{\prime}-t\right)\right] \frac{d T_{f}\left(t^{\prime}\right)}{d t^{\prime}} d t^{\prime}$

So far the solution is general and does not depend on the functional form of $T_{f}$ provided it has a reasonable well defined derivative. Eqn. $\left(16^{\prime}\right)$ shows the steel temperature follows the ISO standard curve $T_{f}$ but lags from it by the amount given by the integral term. This is a convolution of the derivative of the ISO curve and an exponential function. The weighting function of the derivative equals unity at time $t$ and decays exponentially towards earlier times. Substituting the ISO 834 standard exposure curve, eqn. (1), into eqn. (16') and integrating results into

$$
\begin{aligned}
T(t) / T_{C}= & \ln \left(1+t / t_{0}\right)-\exp \left[-A\left(t+t_{0}\right)\right] \\
& \left\{E i\left[A\left(t+t_{0}\right)\right]-E_{i}\left[A t_{0}\right]\right\}
\end{aligned}
$$


where the exponential integral function $\mathrm{Ei}(z)$ is given by a Cauchy principal value integral [1]

$E i(z)={ }_{-\infty}\left(z e^{t} d t / t(z>0)\right.$

The first term in eqn. (17) is the ISO 834 fire test curve. The second term on the right hand describes the lag of the change of temperature caused by the heat capacity of steel. For long times the variable $A\left(t+t_{0}\right)$ grows big compared to unity. Then using the asymptotic expansion of the exponential integral for big $z$ [1]

$E i(z) \rightarrow e^{z} / z$

the temperature lag is expressed

$T_{C} \exp \left[-A\left(t+t_{0}\right)\right]\left\{\exp \left[A\left(t+t_{0}\right)\right] /\left[A\left(t+t_{0}\right)\right]-E i\left(A t_{0}\right)\right\}=$

$T_{C}\left\{A\left(t+\frac{1}{\left.+t_{0}\right)}-\exp \left[-A\left(t+t_{0}\right)\right] E i\left(A t_{0}\right)\right\} \rightarrow 0\right.$

Thus the temperature lag disappears for long fire exposures, because the logarithmic ISO 834 curve time dependence becomes rather flat.

HEATING OF INSULATED STEEL

The general solution of eqn. (9) with the initial condition (4) is very similar to the solution of eqn. $\left(8^{\prime}\right)$. The general solution for arbitrary function $T_{f}(t)$ is

$T(t)=-B T_{f}(t)+A(1+B){ }_{0} t^{t} \exp \left[A\left(t^{\prime}-t\right)\right] T_{f}\left(t^{\prime}\right) d t^{\prime}$

which again can be cast into a "perturbation expansion"

$T(t)=T_{f}(t)-(1+B) \int_{0}^{t} \exp \left[A\left(t^{\prime}-t\right)\right] \frac{d T_{f}\left(t^{\prime}\right)}{d t^{\prime}} d t^{\prime}$

This solution differs from eqn. (16') only by the multiplying factor $1+B$ in front of the integral on the right hand side. This allows for easy interpretation: If $B=0$ eqn. (9) becomes indentical with eqn. ( $\left.8^{\prime}\right)$ and the solution (21) with eqn. $\left(16^{\prime}\right)$ as it should. For positive $B$ the temperature lag, which the latter part of the solution represents, increases by a multiplicative factor.

Substituting into eqn. (21) the explicit formula for the Iso standard exposure curve yields for the steel temperature $T(t)$ a closed form formula

$$
\begin{aligned}
T(t) / T_{C}= & \ln \left(1+t / t_{0}\right)-(1+B) \exp \left[-A\left(t+t_{0}\right]\right. \\
& \left\{E i\left[A\left(t+t_{0}\right)\right]-E i\left[A t_{0}\right]\right\}
\end{aligned}
$$


This is the same as eqn. (10) despite the factor $1+B$ in front of the latter part. Calculating numerical values of $b$ using eqns. (10) through (15) shows it is small $(<0.5)$. However, in the beginning of the curve, where the latter part is dominant, nonzero B drops steel temperatures fast. Thus, negative values of temperature change occur in the beginning as noted earlier, $[3,4]$. For insulated steel the relevant protection time is of the order of one hour. This method is not suitable for calculating the temperatures during the first minutes, but applies well for longer times.

There are two possible ways of using this theoretical result: (i) Theoretically eqns. (10) - (15) determine the constants $A$ and $B$ from first principles, i.e. from known dimensions and material properties of the system. Thus an iteration process using these equations, as will be shown, produces values of $A$ and $B$. (ii) Since this is only a rough model, it is not for granted the prediction of temperatures is accurate enough. Although the gross features of the temperature are right, detailed quantitative determination might be too coarse. Therefore, one could use experimental temperature data and try to determine effective material parameters to be used for design purposes. More work is needed to show, which one of these methods turns out practical.

\section{APPLICATION FOR INSILATION DESIGN}

In fig. 2 the temperatures predicted by eqn. (17) are plotted as a function of time (expressed now in minutes) and the constant $A$ as a parameter. Using the recommendations of ECCS [6] to calculate $h$, and using maximum air and steel temperatures we got somewhat conservative temperature predictions as shown in table 1.

TABLE 1. Comparison of temnerature predictions

\begin{tabular}{|l|l|c|c|c|}
\hline \multirow{2}{*}{ Variable } & Dimension & \multicolumn{3}{|c|}{ Time, min } \\
\cline { 2 - 5 } & & 30 & 60 & 30 \\
\hline Section factor & $\mathrm{m}^{-1}$ & 30 & 10 & 10 \\
A & $\mathrm{s}^{-1}$ & $7.1 \cdot 10^{-4}$ & $2.8 \cdot 10^{-4}$ & $3.2 \cdot 10^{-4}$ \\
$\begin{array}{l}\text { Temperature: } \\
\text { this work }\end{array}$ & $0 \mathrm{C}$ & 510 & 530 & 750 \\
Ref. [6] & & 484 & 467 & 678 \\
\hline
\end{tabular}




\section{STEEL TEMPERATURES}

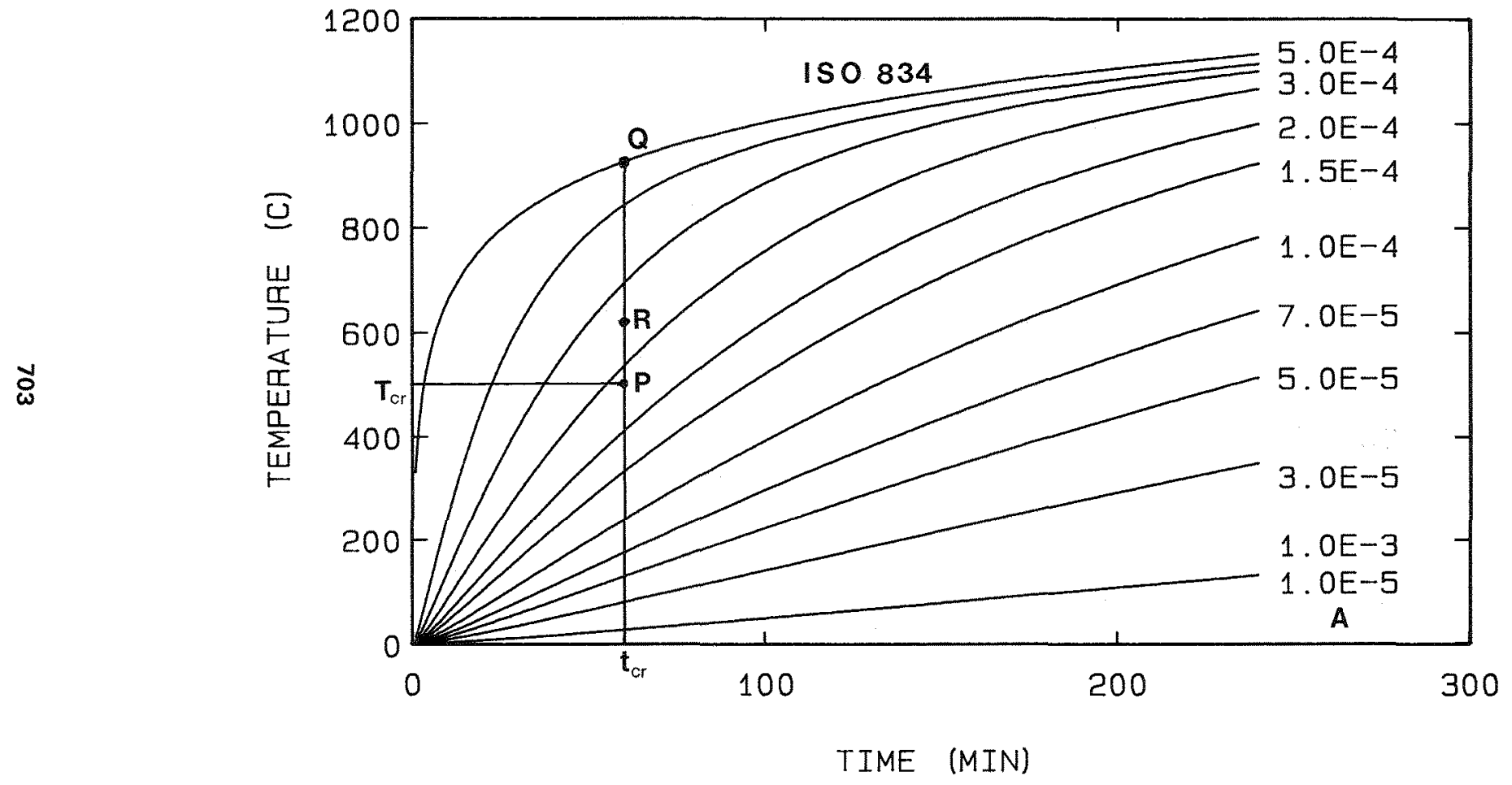

FIGURE 2. Dependence of steel temperatures on time and parameter $A\left(s^{-1}\right)$. For determination of $B$, see text. 
In reality, $h$ is during the heating period smaller than this maximum value. Using some smaller values for $h$, the difference between both theoretical results can be compensated. No comparison with experimental results was tried. This example also shows that the heating of bare steel depends delicately on heat transfer.

For calculation of cladding thickness of insulated steel we carry out an example where the temperature should remain below the critical temperature $\mathrm{T}_{\mathrm{C}}=500{ }^{\circ} \mathrm{C}$ for at least 1 hour. We presume the insulation to be dry material with thermal parameters: density $200 \mathrm{~kg} / \mathrm{m}^{3}$, specific heat capacity $700 \mathrm{~J} / \mathrm{kgK}$ and thermal conductivity $0.2 \mathrm{~W} / \mathrm{km}$. The section factor of the steel is $100 \mathrm{~m}^{-1}$. From eqn. (12) we calculate $\mathrm{Jd}=0.37 \mathrm{~m}$. From fig. 2 we see for time $t_{C r}=1 \mathrm{~h}$ and temperature $T_{C r}=500{ }^{0} \mathrm{C}$ (point $\mathrm{P}$ ), that $A \approx 2.6 \cdot 10^{-4} \mathrm{~s}$. Substituting this into eqn. (10) and taking $N=3$ and $\gamma=\infty$, an approximate $d \approx 15 \mathrm{~mm}$ is obtained. Then $\mathrm{J} \approx 25$ and from eqn. (11), where $b=0.5, B \approx 0.0066$. This is so smal1 that there is no use to continue iteration because the accuracy of fig. 2 is not enough.

In case $B$ were bigger, one should determine a point $R$ along line $P \cap$ such that the calculated $B$ is given through the ratio

$B=P R / O R$

and the new $A$ is the value of the curve going through point $R$. New thickness $d$ is then obtained from eqn. (10). This iteration is continued as long as the coefficients $A$ and $B$ change considerably. In the above example one iteration was sufficient resulting $\mathrm{d} \approx 15 \mathrm{~mm}$ as compared to value $d \approx 16 \mathrm{~mm}$ obtained in ref. [6]. Since $B$ is usually small $(<0.2)$, the method converges quickly.

\section{CONCLUSIONS}

The closed form formulas for the temperatures of unprotected steel, eqn. (17), as well as the solution, eqn. (22), of the approximate equation of the insulated steel can be utilized for fast calculations of steel temperatures. The latter equation suits for quick determination of insulation thickness, because it is mainly a one-parameter (A) function. The dependence on $B$ is weak and is used for fine adjustment. These equations are easily programmed on PC-level microcomputers and allow for iterative determination of any one parameter when others are known.

Eqns. (17) and (22) are useful for deriving analytical fits on experimental data. Theoretical curves present the overall features. Local adjustment to make prediction to coincide with experimental data is a way to determine effective material parameters.

For numerical calculations tabulations of the exponential integral function, eqn. (18), are available, e.g. [1]. The mathematical subroutine libraries on most main frame computers include $E i(z)$. It can also be calculated from simple algorithms $[1,5]$. 


\section{REFERENCES}

1. Abramowitz M., and Stegun, I. A. Handbook of Mathematical Functions, pp. 228 - 230, Dover, New York, 1970.

2. Wickström, U., "Temperature Analys is of Heavily-insulated Steel Structures Exposed to Fire", Fire Safety J. , 9:281 - 285, 1985.

3. Melinek, S. J., and Thomas, P. H., "Heat Flow to Insulated Steel", Fire Safety J., 12:1 - 8, 1987.

4. Bфhm, B., "On Thermal Exposure of Protected Steel Structures: Is it possible to apply a constant $d / k$-value for protection with temperature dependent material properties?" Fire Safety J., 8: $73-75$, $1984 / 85$.

5. Cody, W. J., and Thacher, H. C., "Chebyshev Approximations for the Exponential Integral Ei(x)", Mathematics of Computation 23:289 $303,1969$.

6. ECCS. Technical Committee 3 - Fire Safety of Steel Structures, Design Manual on the European Recommendations for the Fire Safety of Steel Structures, First Ed. (1985).

7. ECCS Technical Committee 3, European Recommendations for the Fire Safety of Steel Structures, Elsevier Scientific Publishing Company, Amsterdam, 1983. 
